\title{
Scaling and Time Warping in Time Series Querying
}

\begin{abstract}
The last few years have seen an increasing understanding that Dynamic Time Warping (DTW), a technique that allows local flexibility in aligning time series, is superior to the ubiquitous Euclidean Distance for time series classification, clustering, and indexing. More recently, it has been shown that for some problems, Uniform Scaling (US), a technique that allows global scaling of time series, may just be as important for some problems. In this work, we will demonstrate that for many real world problems, it is necessary to combine both DTW and US to achieve meaningful results. This is particularly true in domains where we must account for the natural variability of human action, including biometrics, query by humming, motion-capture/animation, and handwriting recognition. We introduce the first technique which can handle both DTW and US, and demonstrate its utility and effectiveness on a wide range of problems in industry, medicine, and entertainment.
\end{abstract}

\section{INTRODUCTION}

We propose to query time-series with both the accommodation of a scaling factor and the consideration of timewarping effects. In this section we justify our proposal with some examples.

\subsection{Justifying the Need for Uniform Scaling and DTW}

Because time series are near ubiquitous, and are becoming increasingly prevalent as our ability to capture and store them improves, there is increasing interest in the database community in techniques for efficiently indexing large time series collections $[7,19]$. The last few years has seen a consensus that in most domains, it is necessary to index with invariance to small local misalignments, and Dynamic Time Warping has been shown to be the right tool for this [12, 21, 24]. More recently, it has been forcefully shown that in many domains it is also necessary to index with invari-

Permission to make digital or hard copies of all or part of this work for personal or classroom use is granted without fee provided that copies are not made or distributed for profit or commercial advantage and that copies bear this notice and the full citation on the first page. To copy otherwise, to republish, to post on servers or to redistribute to lists, requires prior specific permission and/or a fee.

Copyright 200X ACM X-XXXXX-XX-X/XX/XX ...\$5.00. ance to global scale [14]. In this work, we argue that for most real world problems, it is necessary to be able to handle both types of distortions simultaneously. In fact, even a casual glance of existing literature confirms this. For example, in query-by-humming systems, it is well understood that we must allow for uniform scaling in addition to DTW. The current solution is to simply do DTW at many resolutions that span the possible range of tempos. For example, Meek and Birmingham [17] reports "We account for the phenomenon of persons reproducing the same tune at different speeds ... allow(ing) for nine tempo mappings". However, repeating the query nine times clearly slows the system down. Furthermore, it is possible that the best match occurs somewhere in-between the nine discrete scalings. In [15], the authors also note that in addition to the local problems handled by DTW, "(people can) perform faster or slower than usual". They again deal with this with multiple scaled queries, achieving reasonable performance only by the use of parallel processing.

Dynamic Time Warping is frequently used as the basis of gait recognition algorithms, but even in this highly structured domain, it is recognized that uniform scaling is also needed. For example, [11] notes "different gait cycles tend to have unequal lengths". In fact, even if we discount human variability, it is well understood that parallax effects from cameras (static or pan-and-tilt) can produce apparent changes in uniform scaling [10].

The need for uniform scaling has been noted in bioinformatics. Moeller-Levet et. al. [5] noted that previous work that addressed only local scaling (with DTW) is inadequate, and they stressed that "(uniform) scaling factors in the expression level hide similar expressions and have to be eliminated or not considered when assessing the similarity between expression profiles" [11].

Finally, the simultaneous need for both uniform scaling and DTW is well understood in the motion-capture and animation community. For example, Pullen and Bregler [20], explaining their motion-capture editing system, noted "we stretch or compress the real data fragments in time by linearly resampling them to make them the same length as the keyframed fragment ...(then do DTW)" . The computational difficulty of dealing with both uniform scaling and DTW at the same time has even led to practitioners abandoning temporal information altogether! Campbell and Bobick [3] used a phase space representation in which the velocity dimensions are removed, thus completely disregarding the time component of the data. This makes the learning and matching of motion patterns simpler and faster, but 
only at the cost of a massive increase in false positives.

Let us call "DTW with Uniform Scaling" SWM, which stands for Scaled and Warped Matching. In this paper, we study the combined effects of scaling and time-warping in time series querying.

\subsection{Motivating Examples}

Below, we present two concrete examples that require SWM to produce meaningful and intuitive results.
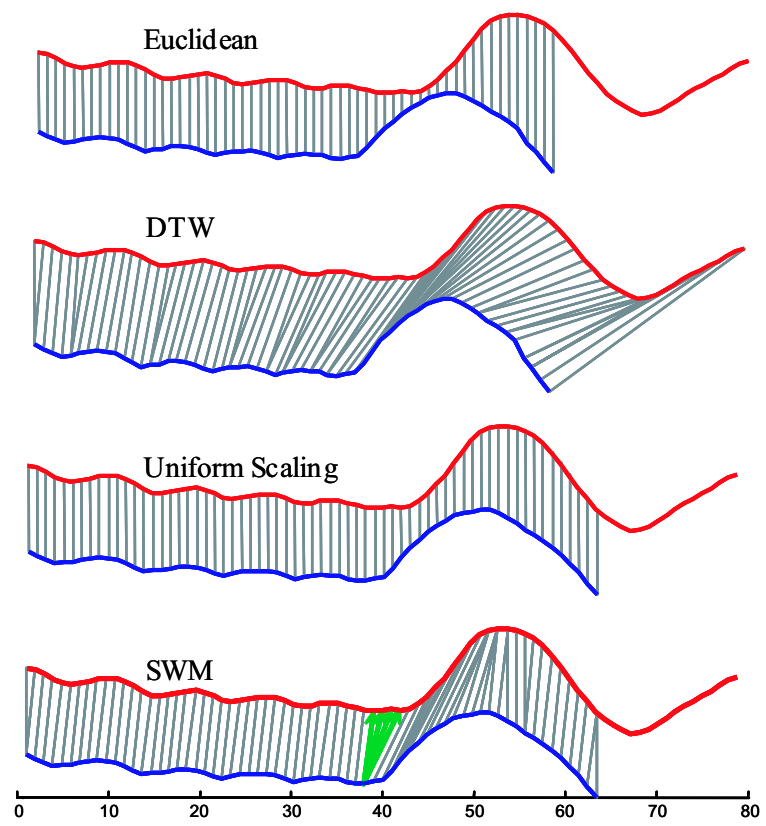

Figure 1: Two examples of an athlete's trajectories aligned with various measures

EXAMPLE 1 (INDEXING VIDEO). There is increasing interest in indexing sports data, both from sports fans who may wish to find particular types of shots or moves, and from coaches who are interested in analyzing their athletes performance over time. As a concrete example, we consider high jump. We can automatically collect the athlete's center of mass information from video and convert into a time series (It is possible to correct for the cameras pan and tilt; see [6]). We found that when we issued queries to a database of high jumps, we only got intuitive answers when doing SWM. It is easy to see why if we look at two particular examples from the same athlete and consider all possible matching options, as shown in Figure 1. From top to bottom:

- If we attempt simple Euclidean matching (after truncating the longer sequence), we get a large error because we are mapping part of the flight of one sequence to the takeoff drive in the other.

- If we simply use DTW to match the entire sequences, we get a large error because we are trying to explain part of the sequence in one attempt (the bounce from the mat) that simply does not exist in the other sequence.

- If we attempt just uniform scaling, we get the best match when we stretch the shorter sequence by $112 \%$.
However the local alignment, particularly of the takeoff drive and up-fight, is quite poor.

- Finally, when we match the two sequences with SWM, we get an intuitive alignment between the two sequences. The global stretching (once again at $112 \%$ ) allows DTW to align the small local differences. In this case, the fact that DTW needed to map a single point in a time series onto 4 points in the other time series suggests an important local difference in one of these sequences. Inspection of the original videos by a professional coach suggests that the athlete misjudged his approach and attempted a clumsy correction just before his takeoff drive.

EXAmPle 2 (QueRY BY Humming). The need for both local and global alignment when working with music has been extensively demonstrated [4, 16, 17, 24]. For completeness, we will briefly review it here. Finding similar sequences of music has applications in copyright infringement detection, analyzing the evolution of music styles [4], automatic annotation, etc. (It is interesting to note that these studies are not confined to human endeavors; similar techniques have been used in animal "music", especially in humpback whales and songbirds [16]). However, the vast majority of research in this area is used to support query by humming.

The basic idea of query by humming is to allow users to search large music collections by providing an example of the desired content, by humming (or singing, or tapping) a snippet. Clearly, humans cannot be expected to reproduce an exact fragment of a song, so the system must be invariant to certain distortions. Some of these are trivial to deal with. For example, the query can be made invariant to key by normalizing both the query and the database to a standard key. However, two types of errors are more difficult to deal with; users may perform the query at the wrong tempo, and users may insert or delete notes. The former corresponds with uniform scaling, the latter with DTW. The music retrieval community has traditionally dealt with these two problems in two ways. The first is to do DTW multiple times, at different scalings [17]. However, this clearly produces scalability problems. The other common approach is to only do DTW with relatively short song snippets as queries believing that short sequences are less sensitive to uniform scaling problems than long sequences. While this is undoubtedly true, short snippets also have less discriminating power.

In Figure 2, we demonstrate the problems with the universally familiar piece of music, Happy Birthday to You. For clarity of illustration, the music was produced by the third author on a keyboard and converted into a pitch contour, however, similar remarks apply to other music representations. From top to bottom:

- Because the query sequence was performed at a much faster tempo, direct application of DTW fails to produce an intuitive alignment.

- Rescaling the shorter performance by a scaling factor of 1.54 seems to improve the alignment, but note for example that the higher pitched note produced on the third "birth..." of the candidate is forced to align with the lower note of the third "happy..." in the query. 
- Only the application of both uniform scaling and DTW produces the correct alignment.

Having increased the readers intuition for DTW and US, and having demonstrated the need to handle both types of distortions simultaneously, we will next define the problem of similarity measurement under SWM more formally.
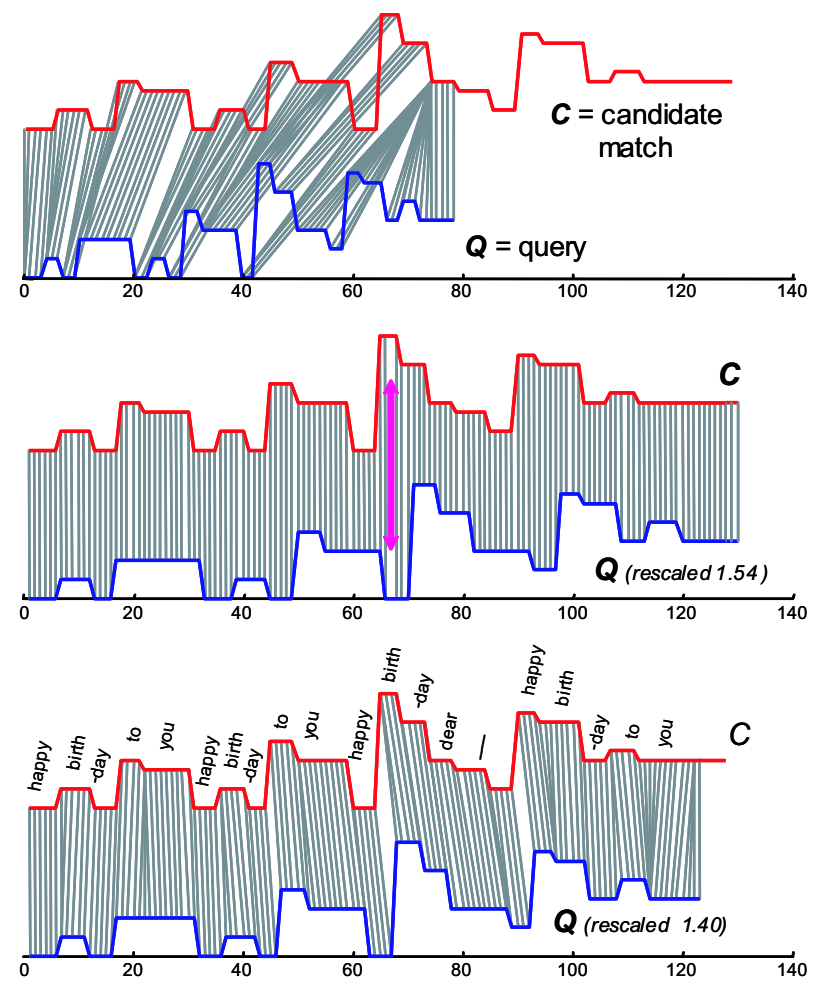

Figure 2: Two performances of Happy Birthday to You aligned with different metrics. Both performances were performed in the same key, but are shifted in the $\mathbf{Y}$-axis for visual clarity.

\section{PROBLEM DEFINITION}

Assume that we are given a database $D$ which contains $M$ time series of length $n$ (Note that this formulation does not preclude the subsequence matching case, or the case where the sequences may be of different lengths, since both cases may be trivially transformed into this formulation). Further, assume that we are given a query $Q$, and a tuple $S F$, which represents the maximum allowable shrinking and stretching of the query length, $S F=\{s, l\}$, where $0<s \leq 1 \leq l<\infty$. Hence the query can be shortened by a factor of up to $s$ or lengthen by a factor of up to $l$. Note that while $s$ is bounded below by zero, and $l$ is bounded from above by infinity, such loose bounds would allow pathological solutions to certain problems, and in any case are surely impossible to support efficiently. We therefore restrict our attention to scaling factors in the range $0.5<s \leq 1 \leq l<2$. Note that this range encompasses the necessary flexibility documented in virtually every domain we are aware of. For instance, in [17], the authors reported excellent results with a query-by-humming system that allows " a (maximum) tempo scaling of 1.25."

Suppose the query sequence $Q=Q_{1}, Q_{2}, \cdots, Q_{m}$. We are interested in tackling the following problems.
Problem 1. Finding the best match to $Q$ in database, for any rescaled version of $Q$ in the range, under the Dynamic Time Warping distance. This problem has never been considered in the literature before.

Problem 2. Assume the data sequences can be longer than the query sequence $Q$. Find the best subsequence among all data sequences that can best match $Q$ with the consideration of both the rescaling factor and dynamic time warping. This problem is realistic in applications such as query by humming. Problem 1 can be seen as a special case where the subsequences are always the full sequences.

Our solution can also solve the following problem although it will not be the focus of our study.

Problem 3. Finding the best match to $Q$ in database, for any rescaled version of $Q$, under the squared Euclidean distance. While this problem has been investigated before [14], our modification reduces the size of the index by half and allows the user to specify the range of scaling without modifying the index.

OBSERVATION 1. For most domains, it is natural for the user to think of the query shrinking and stretching to fit a template. For example, [17] notes that in their experience, amateur singers can speed up their rendition of a song by as much as $200 \%$ or slow down to as little as 50\%. However, for both ease of exposition and ease of implementation, it is much simpler to think of the query as only stretching (or shrinking; this is an arbitrary choice). We can achieve this very easily. Upon receiving a query with accompanying scaling factors, we immediately rescale it such that its length is sm. This is the shortest sequence that the query can shrink into. Note that the longest sequence that the query can be stretched into is $\mathrm{lm}$.

Given this, we only need to implement techniques that consider stretching of the query, then shrinking is implicitly taken care of. In particular, after this rescaling, the new value of $l$ has increased to $l^{*}=l / s$, and the new value of $s$ is then set to $s^{*}=1$. For the rest of this paper, we will make this simplifying assumption in our description and equations; however, when reporting a matching sequence back to the user, we rescale the answer back to the original scaling units.

Given $N$ variable-length data sequences and a query sequence $Q$, we would like to find all subsequences of the data sequences that are "similar" to $Q$. We shall review time warping distance as our similarily measure in the next section.

\section{PRELIMINARIES}

In this section, we review separately time-series querying with time-warping distance and also querying with the scaling effect. For each case, we can apply a lower bounding technique for pruning the search space.

\subsection{Time Warping Distance}

Intuitively, dynamic time warping is a distance measure that allows time series to be locally stretched or shrunk before applying the base distance measure. Definition 1 formally defines time warping distance. 
Definition 1 (Time Warping Distance). Given two sequences $C=C_{1}, C_{2}, \cdots, C_{n}$ and $Q=Q_{1}, Q_{2}, \cdots, Q_{m}$, the time warping distance $D_{t w}$ is defined recursively as follows:

$$
\begin{aligned}
D_{t w}(\phi, \phi)= & 0 \\
D_{t w}(C, \phi)= & D_{t w}(\phi, Q)=\infty \\
D_{t w}(C, Q)= & D_{b a s e}(\operatorname{First}(C), \operatorname{First}(Q))+ \\
& \min \left\{\begin{array}{l}
D_{t w}(C, \operatorname{Rest}(Q)) \\
D_{t w}(\operatorname{Rest}(C), Q) \\
D_{t w}(\operatorname{Rest}(C), \operatorname{Rest}(Q))
\end{array}\right.
\end{aligned}
$$

where $\operatorname{First}(C)=C_{1}$, Rest $(C)=C_{2}, C_{3}, \cdots, C_{n}$, and $D_{b a s e}$ denotes the distance between two entries.

Several metrics were used as the $D_{b a s e}$ distance in previous literature, such as Manhattan Distance [23] and squared Euclidean Distance [12, 22]. We will use squared Euclidean Distance as the $D_{b a s e}$ measure. That is,

$$
D_{b a s e}\left(C_{i}, Q_{j}\right)=\left(C_{i}-Q_{j}\right)^{2}
$$

It is well known that dynamic time warping distance can be computed by filling a warping matrix using a dynamic programming algorithm directly derived from the definition of time warping distance. A warping path can be identified by tracing the elements in the warping matrix that were used to compute the time warping distance. Formally, a warping path $W$ for two sequences $Q$ and $C$ is a sequence of elements $w_{k}=(i, j)_{k}$ in the warping matrix, where $\max (|Q|,|C|) \leq$ $|W| \leq|Q|+|C|-1 .^{1}$

\subsection{Constraints and Lower Bounding}

Keogh [12] suggested a lower bounding measure based on the global constraints on time warping. Recently it has been forcefully shown that for nearly all types of time series data, using an appropriate global constraints always improves the classification or clustering accuracy and the precision and recall of indexing [21]. Therefore a global constraint is typically enforced to limit the warping path to a roughly diagonal portion of the warping matrix.

Two commonly used global constraints exist. The SakoeChiba Band [22] limits the warping path to a band enclosed by two straight lines that are parallel to the diagonal of the warping matrix. The Itakura Parallelogram [9] limits the warping path to be within a parallelogram whose major diagonal is the diagonal of the warping matrix.

Keogh [12] viewed a global constraint as a constraint on the warping path entry $w_{k}=(i, j)_{k}$ and gave a general form of global constraints in terms of inequalities on the indices to the elements in the warping matrix,

$$
j-r \leq i \leq j+r
$$

where $r$ is a constant for the Sakoe-Chiba Band and $r$ is a function of $i$ for the Itakura Parallelogram.

The upper bounding sequence $U W$ and the lower bounding sequence $L W$ of a sequence $Q$ are defined using the term $r$ as follows.

Definition 2 (Enveloping Sequences by Keogh). Let $U W=U W_{1}, U W_{2}, \cdots, U W_{m}$ and $L W=L W_{1}, L W_{2}, \cdots, L W_{m}$,

$$
\begin{aligned}
U W_{i} & =\max \left(Q_{i-r}, \ldots, Q_{i+r}\right) \text { and } \\
L W_{i} & =\min \left(Q_{i-r}, \ldots, Q_{i+r}\right)
\end{aligned}
$$

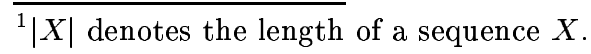

Considering the boundary cases, the above can be rewritten as

$$
\begin{aligned}
U W_{i} & =\max \left(Q_{\max (1, i-r)}, \ldots, Q_{\min (i+r, m)}\right) \text { and } \\
L W_{i} & =\min \left(Q_{\max (1, i-r)}, \ldots, Q_{\min (i+r, m)}\right)
\end{aligned}
$$

These two sequences form an envelope which encloses the sequence $Q$.
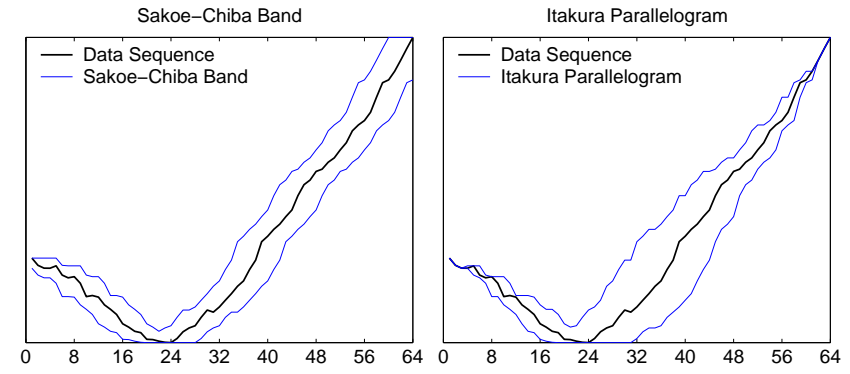

Figure 3: Enveloping sequences derived from two different constraints

The lower bounding measure by Keogh approximates the time warping distance between two sequences $Q$ and $C$ by the Euclidean distance between $C$ and the envelope of $Q$. Equation (1) below formally defines the lower bounding distance by Keogh.

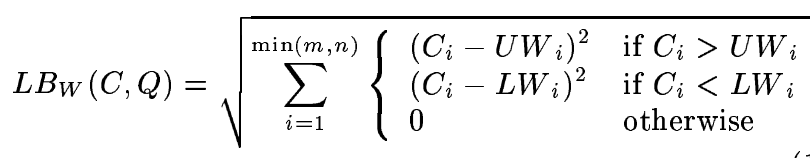

\subsection{Uniform Scaling}

Consider a query sequence $Q=Q_{1}, \cdots, Q_{m}$ and a candidate sequence $C=C_{1}, \cdots, C_{n}$.

We assume $m$ is not greater ${ }^{2}$ than $n(m \leq n)$; hence, the query is typically shorter than the candidate sequence.

If the distance function we use for matching is defined only for two sequences of the same length then we can have the following choices:

1. Truncate $C$ so that we compare $Q$ with $C_{1}, \cdots, C_{m}$

2. Stretch $Q$ to be of length $n$

3. More generally, stretch $Q$ to be of length $p(m \leq p \leq$ $n$ ) and truncate the last $n-p$ values of $C$

Cases 2 and 3 above actually correspond to scaling of the query. We assume that the query can be stretched by a factor of at most $l, l \geq 1$, and can be shrunk by a factor of at most $s, s \leq 1$. Hence, if the query has length $m$, after the scaling, it can be of any length between $s m$ and $l m$.

In order to scale time series $Q=Q_{1}, \cdots, Q_{m}$ to produce a new time series $Q P=Q P_{1}, \cdots, Q P_{p}$ of length $p$, we use the formula

$$
Q P_{j}=Q_{j * m / p} \text { where } 1 \leq j \leq p
$$

Since $p=s m$,

$$
Q P_{j}=Q_{j / s} \text { where } 1 \leq j \leq p
$$

${ }^{2}$ One can also consider shrinking of query, then $n \leq m$. The choice is again arbitrary. 
Note that the above seems to imply that the subscript of $Q_{i}$ can be a fraction. This is possible if we try to evaluate some interpolation on the values of $Q_{\lfloor i\rfloor}$ and $Q_{\lceil i\rceil}$. Or as in the case of [14], only the subscript $\lceil i\rceil$ is used. However, for the purpose of lower bounds, we can use only the given values in $Q_{1}, \ldots, Q_{m}$. We shall see how to do this later.

\subsubsection{Lower bounding uniform scaling}

Initially, we shrink $Q$ to the length of $p=s m$. Consider the converted query $Q P=Q P_{1}, Q P_{2}, \cdots, Q P_{p}$

This query can be stretched to the length of at most $n=l m$. We create two sequences $U S=U S_{1}, \cdots, U S_{n}$ and $L S=L S_{1}, \cdots, L S_{n}$, such that

$$
\begin{aligned}
U S_{i} & =\max \left(Q P_{\max (1, i * p / n)}, \cdots, Q P_{\min (i, p)}\right) \\
& =\max \left(Q P_{\max (1, i * s m / l m)}, \cdots, Q P_{\min (i, p)}\right) \\
& =\max \left(Q P_{\max (1, i * s / l)}, \cdots, Q P_{\min (i, p)}\right) \\
L S_{i} & =\min \left(Q P_{\max (1, i * p / n)}, \cdots, Q P_{\min (i, p)}\right) \\
& =\min \left(Q P_{\max (1, i * s m / l m)}, \cdots, Q P_{\min (i, p)}\right) \\
& =\min \left(Q P_{\max (1, i * s / l)}, \cdots, Q P_{\min (i, p)}\right)
\end{aligned}
$$

Substituting Equation (2), we get

$$
\begin{aligned}
U S_{i} & =\max \left(Q_{\max (1, i * s / l) / s}, \cdots, Q_{\min (i, p) / s}\right) \\
& =\max \left(Q_{\max (1, i / l)}, \cdots, Q_{\min (i / s, m)}\right) \\
L S_{i} & =\min \left(Q_{\max (1, i * s / l) / s}, \cdots, Q_{\min (i, p) / s}\right) \\
& =\min \left(Q_{\max (1, i / l)}, \cdots, Q_{\min (i / s, m)}\right)
\end{aligned}
$$

Now, we can make the bounds more realistic by removing the assumption that we can have a fractional subscript $i$ for an entry $Q_{i}$.

$$
\begin{aligned}
U S_{i} & =\max \left(Q_{\max (1,\lfloor i / l\rfloor)}, \cdots, Q_{\min (\lceil i / s\rceil, m)}\right) \\
L S_{i} & =\min \left(Q_{\max (1,\lfloor i / l\rfloor)}, \cdots, Q_{\min (\lceil i / s\rceil, m)}\right)
\end{aligned}
$$

These sequences bounds the points of the time series $Q$ that can be matched with $C$.

The lower bounding function, which lower bounds the distance between $Q$ and $C$ for any scaling factor SF, $1<S F \leq$ $S F_{\max }$, can now be defined as:

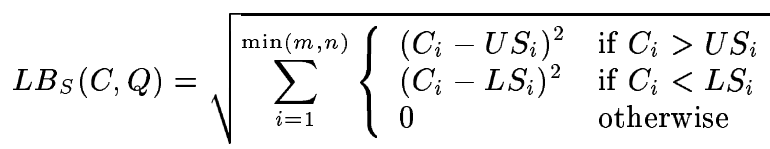

Lemma 1. For any two sequences $Q$ and $C$ of length $m$ and $n$ respectively, the value of $L B_{S}(C, Q)$ lower bounds the distance between $C$ and $Q$ under a scaling of $Q$ between $s$ and $l$, where $s \leq 1, l \geq 1$.

Proof. The matching path $w_{k}=(i, j)_{k}$ defines a mapping between the indices $i$ and $j$. Each such mapping constitutes a term $\left(C_{i}-Q_{j}\right)^{2}$ to the required distance. We will show that each term $t_{l b}$ in the square root of our lower bounding distance $L B_{S}(C, Q)$ can be matched with a term $t$ resulted from the one-to-one mapping, with $t_{l b} \leq t$.

Since $s j \leq i \leq l j \Rightarrow i / l \leq j \leq i / s$ and by definition

$$
\begin{aligned}
U S_{i} & =\max \left(Q_{\max (1,\lfloor i / l\rfloor)}, \cdots, Q_{\min (\lceil i / s\rceil, m)}\right) \\
L S_{i} & =\min \left(Q_{\max (1,\lfloor i / l\rfloor)}, \cdots, Q_{\min (\lceil i / s\rceil, m)}\right)
\end{aligned}
$$

thus $U S_{i}=\max \left(Q_{\max (1,\lfloor i / l\rfloor)}, \cdots, Q_{j}, \cdots, Q_{\min (\lceil i / s\rceil, m)}\right) \geq$ $Q_{j}$, or

$$
C_{i}-U S_{i} \leq C_{i}-Q_{j}
$$

If $C_{i}>U S_{i}$ then $C_{i}-U S_{i}>0$, hence

$$
\left(C_{i}-U S_{i}\right)^{2} \leq\left(C_{i}-Q_{j}\right)^{2}
$$

Similarly we can show that if $C_{i}<L S_{i}$ then

$$
\left(C_{i}-L S_{i}\right)^{2} \leq\left(C_{i}-Q_{j}\right)^{2}
$$

\section{SCALING AND TIME WARPING}

Having reviewed time warping, lower bounding and uniform scaling, this section introduces scaling and time warping (SWM).

We are interested in being able to scale the query and also to find nearest neighbor or evaluate range query by means of time-warping distance. As noted in [14], a naïve search for the uniform scaling problem alone requires $O(|D| *$ $(m-n))$ time, where $m-n$ is the range of lengths resulting from scaling. Time-warping computation alone also requires $O\left(n^{2}\right)$ time for time-series length of $n$. Hence we need to find a more efficient technique for the SWM problem.
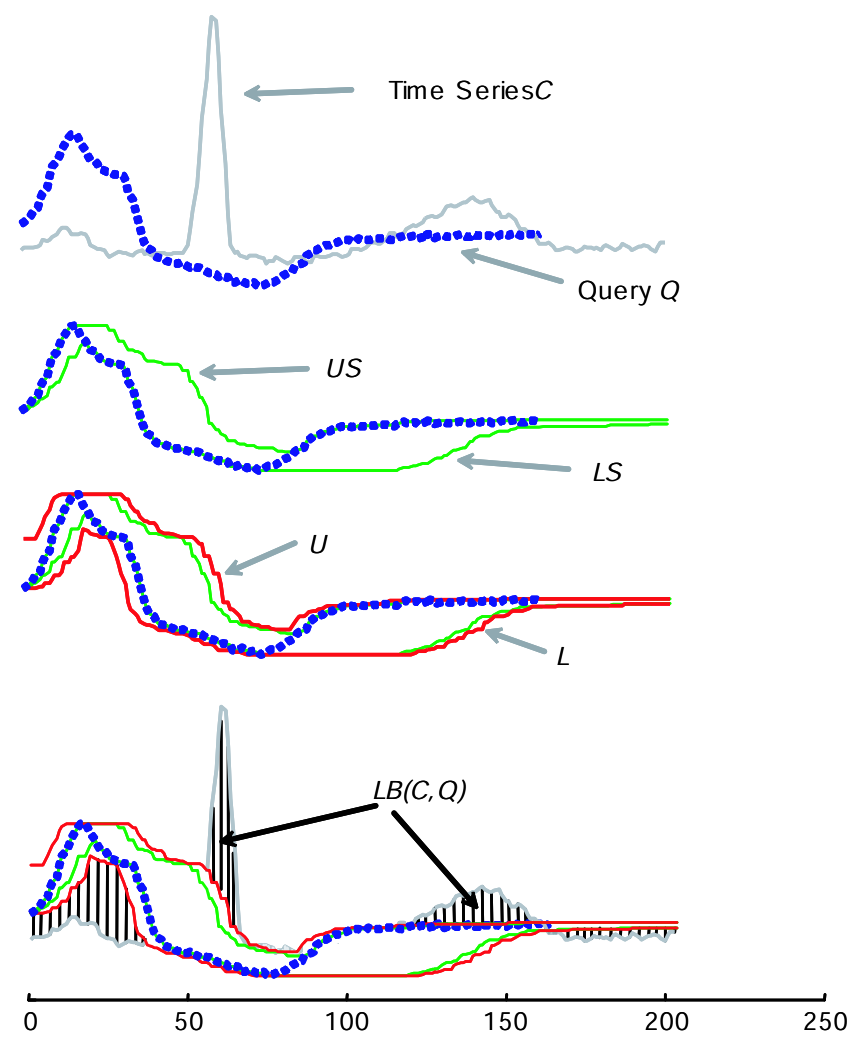

Figure 4: An illustration of the SWM envelopes. From top to bottom: A time series $C$ and a query $Q$; The query $Q$ bounded from above and below respectively by $U S$ and $L S$, the envelope for scaling; The series $U S$ bounded above by $U$ and $L S$ bounded below by $L$, forming the overall envelope for scaling and time warping; and the lower bounding distance LB derived from the overall envelope.

In previous sections, we reviewed the lower bounding technique for each sub-problem. Here, we propose to combine these lower bounds to form overall lower bounds for the 
querying problem. Figure 4 illustrates this graphically. ${ }^{3}$ We apply time warping on top of scaling, i.e. we scale the query first, and then measure the time-warping distance of the scaled query with each candidate sequence. Typically, the time warping with Sakoe-Chiba Band constrains the warping path by a fraction of the query length, which is translated into a constant $r$. Hence, if the fraction is $10 \%$, then $r=0.1|Q|$. If the length of $Q$ is changed according to the scaling fraction $\rho$, that is, $Q$ is changed to $\rho Q$, then the Sakoe-Chiba constraint is $r=0.1|\rho Q|$. Hence, we have $r=r^{\prime} \rho$, where $r^{\prime}$ is the time-warping constraint on the unscaled query, and $\rho$ is the scaling factor.

The lower envelope $L_{i}$ and upper envelope $U_{i}$ on $Q$ can be deduced as follows: Recall that the upper and lower bounds for uniform scaling between $s$ and $l$ is given by the following:

$$
\begin{aligned}
U S_{i} & =\max \left(Q_{\max (1,\lfloor i / l\rfloor)}, \cdots, Q_{\min (\lceil i / s\rceil, m)}\right) \\
L S_{i} & =\min \left(Q_{\max (1,\lfloor i / l\rfloor)}, \cdots, Q_{\min (\lceil i / s\rceil, m)}\right)
\end{aligned}
$$

and the upper and lower bounds for a Sakoe-Chiba Band time-warping constraint factor of $r$ for a query point $Q_{i}$ is given by:

$$
\begin{aligned}
U W_{i} & =\max \left(Q_{\max (1, i-r)}, \cdots, Q_{\min (i+r, m)}\right) \\
L W_{i} & =\min \left(Q_{\max (1, i-r)}, \cdots, Q_{\min (i+r, m)}\right)
\end{aligned}
$$

Therefore, when we apply time-warping on top of scaling the upper and lower bounds will be:

$$
\begin{aligned}
& U_{i}=\max \left(U W_{\max (1,\lfloor i / l\rfloor)}, \cdots, U W_{\min (\lceil i / s\rceil, m)}\right) \\
& =\max \left(Q_{\max \left(1,\lfloor i / l\rfloor-r^{\prime}\right)}, \cdots, Q_{\min \left(\lfloor i / l\rfloor+r^{\prime}, m\right)}, \cdots,\right. \\
& \left.Q_{\max \left(1,\lceil i / s\rceil-r^{\prime}\right)}, \cdots, Q_{\min \left(\lceil i / s\rceil+r^{\prime}, m\right)}\right) \\
& =\max \left(Q_{\max \left(1,\lfloor i / l\rfloor-r^{\prime}\right)}, \cdots, Q_{\min \left(\lceil i / s\rceil+r^{\prime}, m\right)}\right) \\
& L_{i}=\min \left(L W_{\max (1,\lfloor i / l\rfloor)}, \cdots, L W_{\min (\lceil i / s\rceil, m)}\right) \\
& =\min \left(Q_{\max \left(1,\lfloor i / l\rfloor-r^{\prime}\right)}, \cdots, Q_{\min \left(\lfloor i / l\rfloor+r^{\prime}, m\right)}, \cdots,\right. \\
& Q_{\max \left(1,\lceil i / s\rceil-r^{\prime}\right)}, \cdots, Q_{\min \left(\lceil i / s\rceil+r_{s}, m\right)} \\
& =\min \left(Q_{\max \left(1,\lfloor i / l\rfloor-r^{\prime}\right)}, \cdots, Q_{\min \left(\lceil i / s\rceil+r^{\prime}, m\right)}\right)
\end{aligned}
$$

The lower bound function which lower bounds the distance between $Q$ and $C$ for any scaling factor in $S F=s, l$ and time warping with the Sakoe-Chiba Band constraint factor of r' on a given query $Q$ is given by:

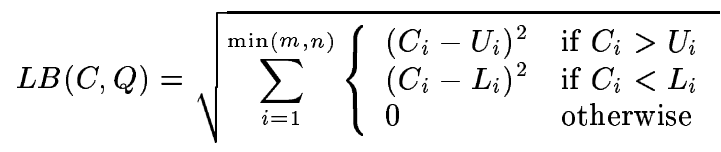

Lemma 2. For any two sequences $Q$ and $C$ of length $m$ and $n$ respectively, given a scaling constraint of $S F=\{s, l\}$ (see Section 2 on problem definition), where $s \leq 1$ and $l \geq$ 1, and a Sakoe-Chiba Band constraint factor of $r^{\prime}$ on the original (unscaled) query $Q$, the value of $L B(C, Q)$ lower bounds the time-warping distance between $C$ and $Q$ under $a$ uniform scaling of $Q$ between $s$ and $l$ and the factor $r^{\prime}$.

\footnotetext{
${ }^{3}$ In this example, the scaling factors are $s=1, l=1.25$; for the warping envelope, $r^{\prime}$ is $3 \%$.
}
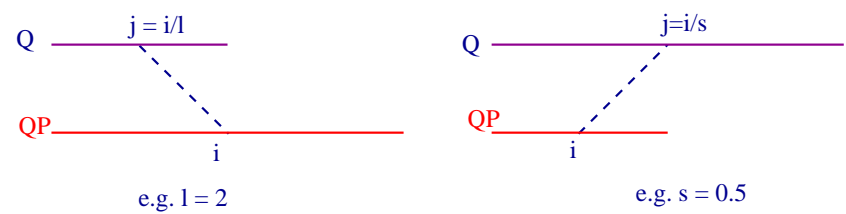

Figure 5: An illustration of the scaling effect, given a query $Q, Q P$ is the result after scaling. Note that the time-warping factor $r^{\prime}$ applies to $Q$. Hence the range of $j$ is given by $i / l-r^{\prime}, \ldots i / s+r^{\prime}$.

PRoOF. The matching warping path $w_{k}=(i, j)_{k}$ defines a mapping between the indices $i$ and $j$. Each such mapping constitutes a term $t=\left(C_{i}-Q_{j}\right)^{2}$ to the required distance. We will show that the $i$-th term $t_{l b}$ in our lower bounding distance $L B(C, Q)$ can be matched with the term $t$ resulting in a one-to-one mapping, with $t_{l b} \leq t$. For the $i$-th term $t_{l b}$, if $C_{i}>U_{i}$, then $t_{l b}=\left(C_{i}-\bar{U}_{i}\right)^{2}$; if $C_{i}<L_{i}$, then $t_{l b}=\left(C_{i}-L_{i}\right)^{2}$, otherwise $t_{l b}=0$, which is always $\leq t$.

For scaling plus time-warping, the effective constraint on the range of $j$ is given by: $i / l-r^{\prime} \leq j \leq i / s+r^{\prime}$ By Equations 4 and 5

$$
\begin{aligned}
& U_{i}=\max \left(Q_{\max \left(1,\lfloor i / l\rfloor-r^{\prime}\right)}, \cdots, Q_{\min \left(\lceil i / s\rceil+r^{\prime}, m\right)}\right. \\
& L_{i}=\min \left(Q_{\max \left(1,\lfloor i / l\rfloor-r^{\prime}\right)}, \cdots, Q_{\min \left(\lceil i / s\rceil+r^{\prime}, m\right)}\right. \\
& \text { thus } U_{i} \geq Q_{j}, \text { or } C_{i}-U_{i} \leq C_{i}-Q_{j}
\end{aligned}
$$

Hence if $\left(C_{i}>U_{i}\right)$ then $C_{i}-U_{i}>0$ and we have

$$
\left(C_{i}-U_{i}\right)^{2} \leq\left(C_{i}-Q_{j}\right)^{2}
$$

Similarly we can show that when $\left(C_{i}<L_{i}\right)$

$$
\left(C_{i}-L_{i}\right)^{2} \leq\left(C_{i}-Q_{j}\right)^{2}
$$

\section{EXPERIMENTS ON PRUNING POWER}

This section describes the experiments carried out to verify the effectiveness of the proposed lower bounding distance. To evaluate the effectiveness of the proposed lower bounding distance, and thus the proposed solution, an objective measure of the quality of a lower bounding distance is required. Keogh defined the Pruning Power $P$ in [12] as follows,

$$
P=\frac{\text { Number of objects that do not require full DTW }}{\text { Number of objects in database }}
$$

The Pruning Power is an objective measure because it is free of implementation bias and choice of underlying spatial index. This measure has become a common metric for evaluating the efficiency of indexing, therefore, it was adopted in evaluating the proposed lower bounding distance.

Extensive experiments were conducted on as many as 41 different datasets. These datasets, which represent time series from different domains, were obtained from "The UCR Time Series Data Mining Archive" [13].

As the datasets came from a wide variety of different domains, they differed significantly in size and in the length of individual data sequences. In order to produce meaningful results, both parameters must be controlled. Thus, from each original dataset, we derived seven sets of data, each containing 1024 data sequences, with variable lengths of $16,32,64,128,256,512$ and 1024 , respecctively. Short sequences were produced by using only prefixes of the original datasets while long sequences were produced by concatenat- 
ing original seqeuences. All experiments were done on these derived datasets.

To compute the pruning power of the proposed lower bounding distance, the 1-nearest neighbor search was performed using the linear-scan algorithm. A random subsequence was chosen from the dataset to act as the query, and the remaining 1023 sequences acted as the data. The search was repeated for 50 trials using a different subsequence as query. The actual dynamic time warping distance did not need to be calculated if the lower bounding measure gave a value larger than the time warping distance of the current nearest neighbor. The fraction of sequences that did not require calculation of actual time warping distance became the pruning power of the lower bounding measure in that query. The average of the 50 queries were reported as the pruning power of that particular dataset.

Unless stated otherwise, in all experiments, the length of data was 1024 data points; the scaling factor was between 1.5 and its reciprocal; the length of query was set so that the longest rescaled query is at most as long as the data; and the width of the Sakoe-Chiba Band was set to $10 \%$ of the length of the query. In fact, recent evidence suggests that this is a pessimistic setting, and real world problems benefit from even tighter constraints [21].

Figure $6^{4}$ shows how the pruning power of the proposed lower bounding measure varies as the lengths of data change on different datasets. For a majority of datasets, the pruning power increased with the length of data, suggesting that the proposed algorithm is likely to perform well in real-life environment, in which long sequences of data are collected for a long period of time. More than $60 \%$ of the datasets obtained a pruning power above $90 \%$. All but two of the datasets exhibited a pruning power of over $60 \%$ at length 1024 . Even at length 16 , over $60 \%$ pruning power was achieved in threefourths of the datasets. Figure 7 shows the pruning power averaged over all datasets; $87 \%$ of data sequences of length 1024 and $65 \%$ of data sequences of length 16 did not require computation of the actual time warping distances.

Figure 8 shows the effect of varying the range of allowed scaling factors on pruning power. Note the $\mathrm{x}$-axis indicates the upper bound range of allowed scaling factor. The lower bound range of allowed scaling factor is the reciprocal of the upper bound. For instance, the label 2.0 indicates that the range of allowed scaling factor is between $1 / 2.0=0.5$ and 2.0. In particular, the label 1.0 indicates that the time warping distance was calculated without scaling. It also implied that the size of the range was not increasing linearly. However, the important observation is that for all sizes of ranges, a pruning power of over $90 \%$ was achieved in nearly three-fourths of the datasets. For almost all datasets, the pruning powers never dropped below $60 \%$.

A more detailed look into the actual data provided some insights as to why most datasets give very high pruning power and why the few other datasets give less than desired pruning power. Figure 9 shows sample sequences from the two datasets that give the lowest pruning power. And

\footnotetext{
${ }^{4} \mathrm{We}$ note that some of the figures in this section suffer from monochromatic reproduction. We encourage the interested reader to visit www.cs.ucr.edu/ eamonn/SIGMOD05anonymous/ for large scale color graphics with additional details. We gratefully acknowledge Dr. Keogh of the UCR time series archive for hosting these files, thus allowing our anonymity.
}

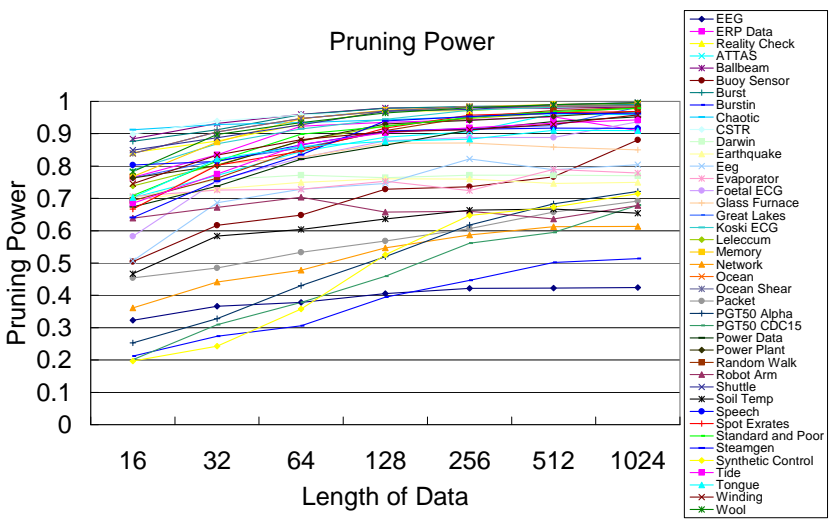

Figure 6: Pruning Power vs. Length of Data

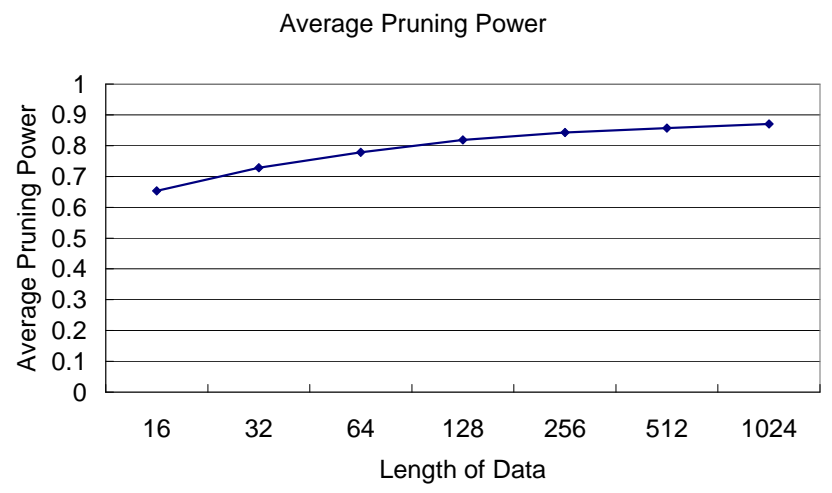

Figure 7: Average Pruning Power vs. Length of Data

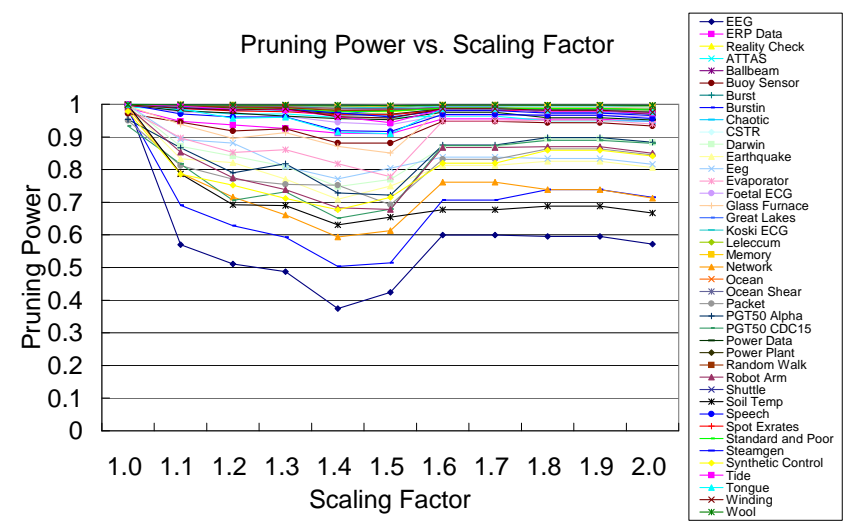

Figure 8: Pruning Power vs. Scaling Factor 

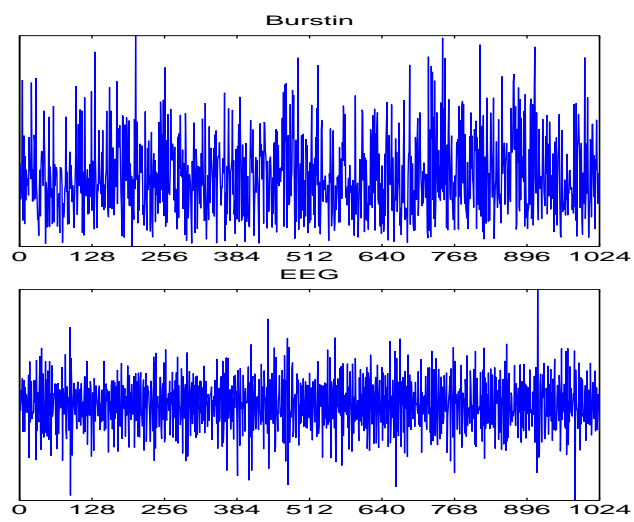

Figure 9: Data Giving the Lowest Pruning Power
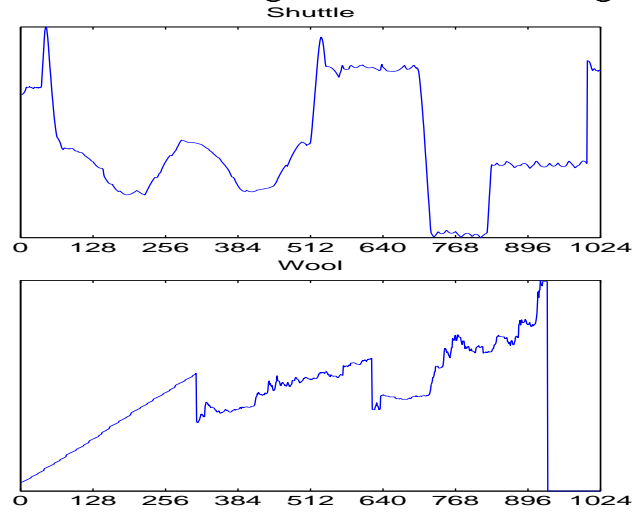

Figure 10: Data Giving the Highest Pruning Power

Figure 10 shows the sample sequences from the two datasets that give the highest pruning power. The difference between them is rather obvious visually. The sequences giving the lowest pruning power are those that fluctuate vigorously. The sequences giving the highest pruning power are those that are rather smooth. The authors conjectured that the smoothness of the data gives the high pruning power and more importantly, the success of the lower bounding techniques.

Nevertheless, we note that vigorously fluctuating datasets are far less common than smooth datasets. Figure 11 illustrates this claim by showing the pruning power averaged over all the datasets, as the range of allowed scaling factor changes. For most ranges of scaling factors, the pruning powers achieved are above $90 \%$.

Interestingly, an obvious trend was observed in all datasets. The pruning power decreased when the range increased from 1.0 to about 1.4. It then climbed up dramatically up to 1.6. Afterwards, the pruning power more or less leveled off. This trend can be seen clearly in Figure 11. The authors suspect that the interesting behavior may be due to the periodicity of many time-series, as illustrated by the synthetic data and query pair shown in Figure 12.

Figure 12 shows a synthetic data $C$ with its envelopes $U$ and $L$. There are also two scaled queries. Query $Q_{2}$ is scaled by a factor of 2. Query $Q_{1.5}$ is the same query scaled by a factor of 1.5. Although both queries do not match well with $C, Q_{2}$ is further from $C$ than $Q_{1.5}$ by a difference represented by the region $X+Z-Y$. This suggests that it

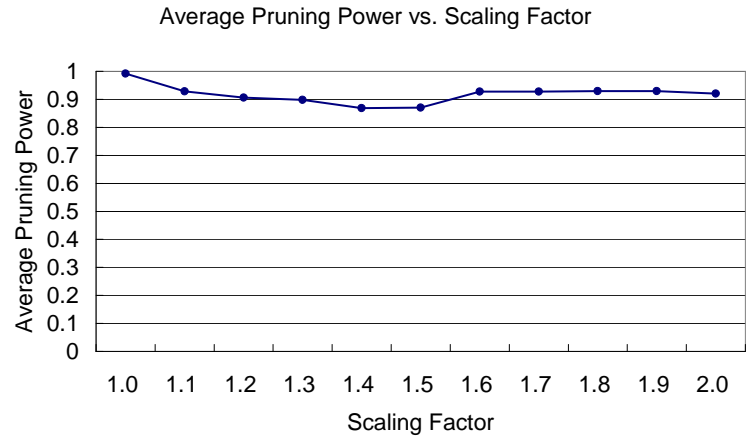

Figure 11: Average Pruning Power vs. Scaling Factor

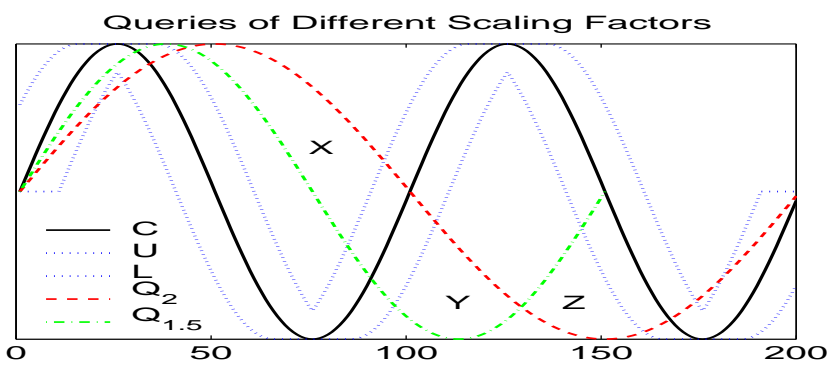

Figure 12: Queries of Different Scaling Factors

is more probable for $Q_{1.5}$ to be included in the candidate set than $Q_{2}$, thus, producing more false alarms. Further studies can be conducted to verify the above claim.

In conclusion, the result shows that the proposed lower bounding measure effectively speeds up query computation. It also confirms the applicability of lower bounding technique, even when a tight lower bound may not be readily obtainable.

\section{INDEXING FOR SWM}

Next we consider Problem 2 in Section 2, which is to find the best matching time series subsequences in the database for a given query. Both scaling and time warping are to be handled at the same time. For this problem, we investigate into the role of indexing in performance enhancement.

\subsection{Related Work}

The following subsections review existing subsequence matching algorithms. These algorithms worked under the Euclidean distance metric.

\subsubsection{Fast subsequence matching in time-series data- bases}

Faloutsos et al. proposed in [7] the now ubiquitous method of indexing time-series subsequences under the Euclidean distance metric. The idea is to place a sliding window on every possible position of every data sequence. For each such placement, a subsequence is extracted. Dimensionality reduction technique such as Discrete Fourier Transform is applied to reduce the subsequence to a feature point in the 
$f$-dimensional space. Feature points from nearby windows are grouped together to span a minimum bounding rectangle (MBR). These MBRs are stored in an ordinary spatial index such as R-Tree [8] and $\mathrm{R}^{*}$-Tree [1]. MBRs are stored instead of individual feature points because the number of feature points can be as high as $O(n l)$ where $n$ is the number of original data sequence and $l$ is the length of each data sequence.

To process a query, the query sequence is divided into consecutive disjoint subsequences. Each subsequence is again transformed to a feature point in the $f$-dimensional space. A range query is performed for each feature point. The union of the results from these range queries form the candidate set. It is proven that if the original tolerance is $\epsilon$ and the query is divided into $p$ subsequences, no false dismissal is generated even if each range query has a tolerance as low as $\epsilon / p$.

\subsubsection{Duality-based subsequence matching in time- series databases}

More recently, Moon et al. [19] proposed another approach for subsequence indexing that is a dual approach to the one described above. This is a dual approach because the roles of the data sequence and the query sequence are exchanged. Instead of storing MBRs, each data sequence is divided into consecutive disjoint subsequences. Each subsequence can be indexed using a spatial index.

In order to guarantee no false dismissal, a sliding window must be placed on a query sequence. Naïvely, a range query can be performed on each subsequence obtained. However, this requires repeated accessing of the index structure, incuring extra page accesses. Consequently, instead of performing range query individually, several subsequences can be grouped together to span an MBR. These MBRs are appropriately enlarged by the user specified tolerance at each dimension. A query is performed based on each resulting enlarged MBR. The union of the results form the candidate set. The actual sequences correponded to the entries in the candidate set are retrieved to compute the actual distance from the query.

The advantage of Moon's approach over Faloutsos' is that individual feature points, instead of MBRs, are stored. It is anticipated that fewer false alarms will be generated because it is less likely that a query region will overlap with the feature points (compared to overlapping with an MBR), as illustrated in Figure 13. Moreover, our enveloping technique on the query translates naturally to query regions (or query MBRs). It is intuitive that enclosing the query regions in an MBR would produce fewer false alarms than enclosing the data with another MBR.

However, since a query may match a data subsequence at any offset but only disjoint subsequences are stored in the database, a query matching a data subsequence may not necessarily contain a subsequence corresponding to the disjoint subsequence. To guarantee the correctness of Moon's approach, a query must be sufficiently long to include at least one disjoint subsequence at every alignment with the data sequences as shown in Figure 14.

Formally, Moon et al. [18, 19] proved the following lemma about the number of disjoint subsequences included by a query of a given length.

Lemma 3. If a sequence $C$ is divided into disjoint subsequences of length $\omega$, the minimum number of disjoint subse-

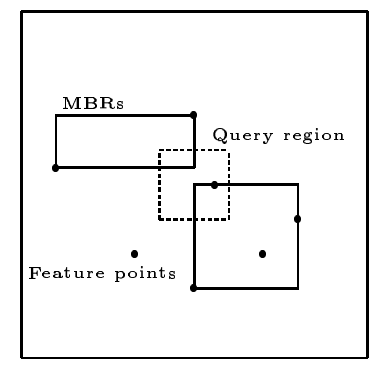

Figure 13: It is less likely that a query region will overlap with the feature points (compared to overlapping with an MBR).

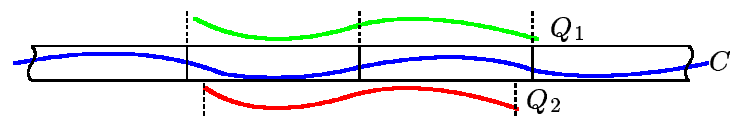

Figure 14: Query must be sufficiently long to include at least one disjoint subsequence at every alignment with the data sequence $C-Q_{1}$ is long enough but $Q_{2}$ is too short.

quences $p$ included by a query of length $l$ is given by

$$
p=\lfloor(l+1) / \omega\rfloor-1
$$

As a consequence of Lemma 3, Moon et al. [18, 19] also proved the following lemma about the relationship between the maximum length of a disjoint subsequence and minimum length of a query holds.

LEMMA 4. If the minimum length of a query sequence is given by $\min (Q)$, then the maximum length of a disjoint subsequence is given by

$$
\lfloor(\min (Q)+1) / 2\rfloor
$$

\subsection{Proposed Indexing for SWM}

This section describes an index that supports scaled and warped matching of subsequences.

In essence, the enveloping technique enables the construction of indices supporting scaled and warped matching (and in general, any measures that do not follow the triangular inequality) by introducing a lower bounding distance that satisfies the triangular inequality. Consequently, almost any spatial access methods such as R-Tree [8], $\mathrm{R}^{*}$-Tree [1], and $\mathrm{X}$-Tree [2] can be used as the underlying indexing structure. Applying different envelopes on the query sequence allows the index to support arbitrarily-defined measures for which the enveloping technique is applicable, such as scaled and warped matching.

\subsubsection{Index construction algorithm}

Algorithm 1 shows the index construction algorithm. Each data sequence is divided into $\lfloor|C| / \omega\rfloor^{5}$ disjoint subsequences each of length $\omega$. These subsequences are inserted into the index structure, together with its ID. Note that only disjoint subsequences are stored, so the index can be kept to a reasonable size. However, these subsequences are stored directly into the index; no extra MBRs are created, leaving the responsibility of grouping feature points back to the underlying spatial access method.

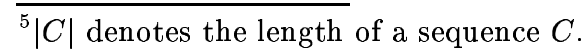




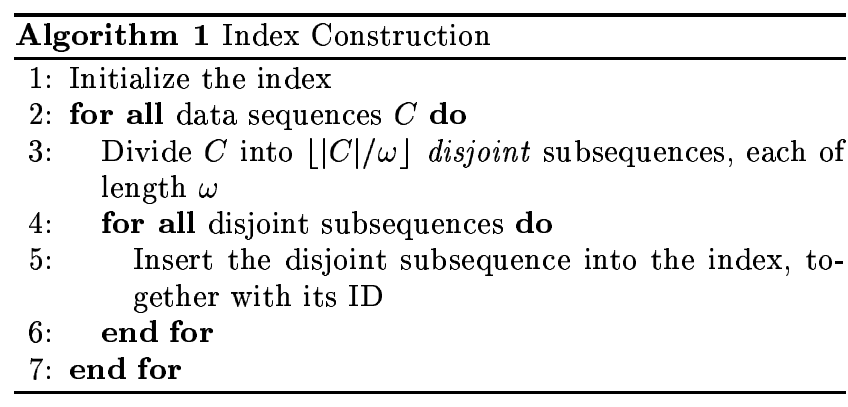

\subsubsection{Utilizing the index}

The key to the Index for Scaled and Warped Matching lies mostly on the query processing algorithm, as shown in Algorithm 2.

Given the query sequence $Q$, the minimum number of disjoint subsequences $p$ included by $Q$ is computed. The lower and upper bounding sequences are then constructed from $Q$. Lower and upper bounding subsequence pairs are then extracted by placing a sliding window on every possible position of the lower and upper bounding sequences. Each pair of subsequences span an MBR. Enlarge the MBRs by the tolerance $\epsilon / \sqrt{p}$. Range queries ${ }^{6}$ are performed using the enlarged MBRs and the results are stored in the candidate set. For each resulting candidate, the actual sequence $C$ is retrieved to perform post-processing step. For each data suffix of $C$, find the best match by trying all possible scalings. The sequence together with the offset and the best scaling factor is returned if the actual distance lies within the given tolerance $\epsilon$.

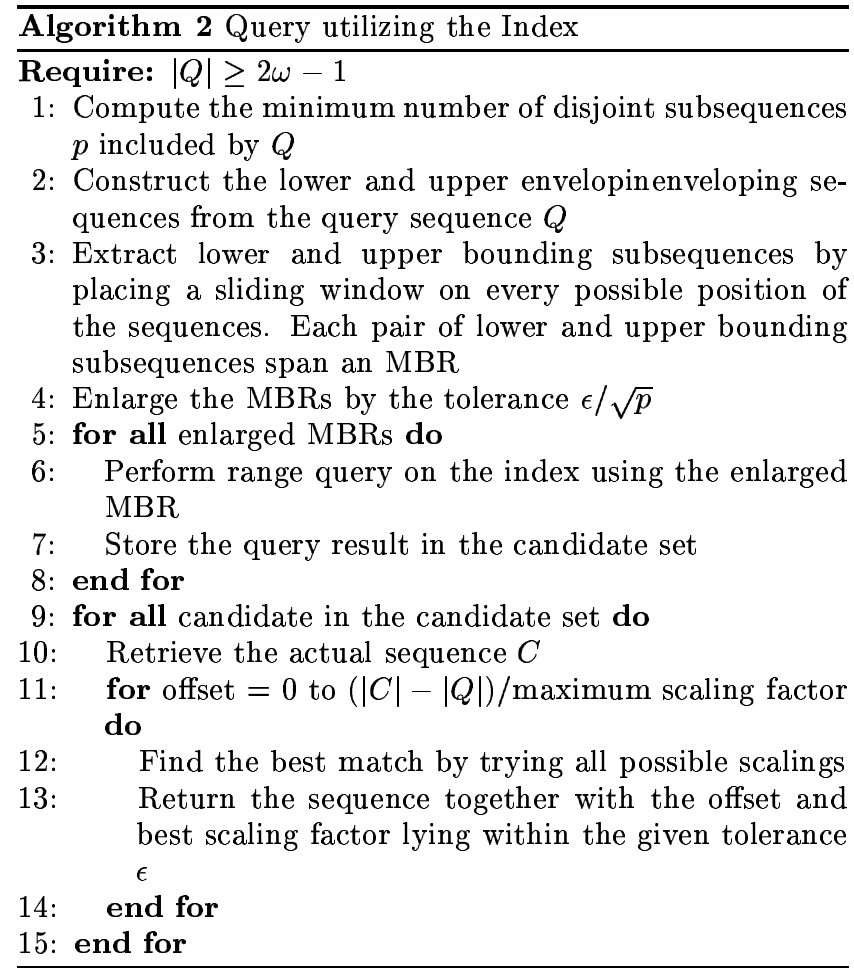

Algorithm 2 returns all the sequences that match the

${ }^{6}$ The bounding box defines a high-dimensional range in search space for locating data. query sequence at a certain offset at a certain scaling factor. However, a large number of range queries are required at line 6 . This can be reduced by grouping several query MBRs to form a larger query MBR.

\subsection{Experiments on Index}

To investigate the effect of using our proposed index, indices were built on the datasets and various queries were issued. For each dataset, queries were issued with the range of $0.001,0.0001$, and 0.00001 . For each range, 50 queries were performed using 50 different subsequences extracted randomly from the data as the query sequences. The average values of the 50 queries were reported for each dataset. Due to lack of space, only the results for the range 0.0001 will be presented. Similar results were obtained using other ranges.

For each query, the number of page access required to produce the results was recorded and compared with the number of page access required if linear scan was performed. Quantitatively, the relative page access is defined as

Relative Page Access =

\section{Page Access Required using an index \\ Page Access Required by linear scan}

Figure 15 shows the relative page access of the datasets versus the length of data. The relative page access varied significantly from approximately less than 0.2 and up to less than 1.8. For short data of length 16 , the relative page access is almost always larger than 1 , suggesting that it is generally not a wise idea to use index for short data. However, as the length of data increases, the relative page access decreases in general, as evident in Figure 16, which shows that the relative page access decreases as the length of data increases. This is an important result for the proposed index, signaling that the index is likely to perform progressively better as the length of data increases.

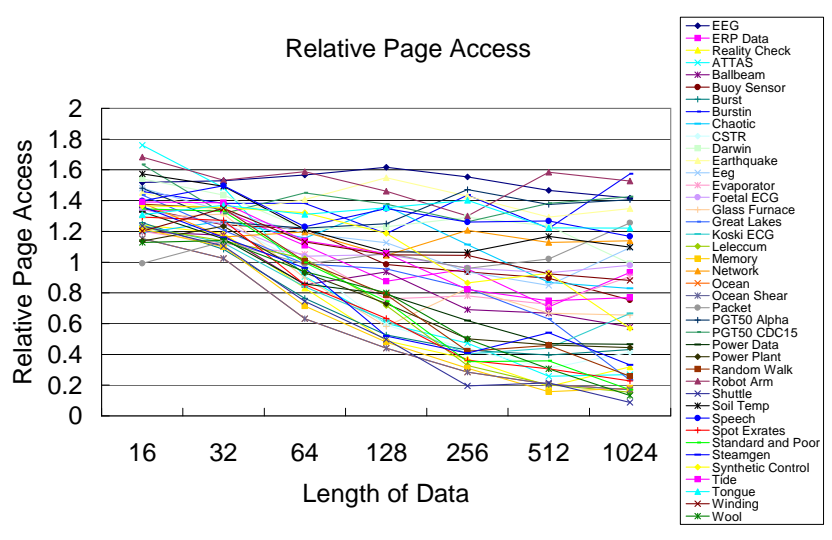

Figure 15: Relative Page Access vs. Length of Data

Figure 16 also shows that the relative page access approaches 1 when the length of data is between 64 and 128 . This suggests that the use of index should be considered if the length of data is greater than 64 . The fact that about half of the datasets achieved a relative page access below 1 at length 64 and that over $70 \%$ of the datasets achieved 


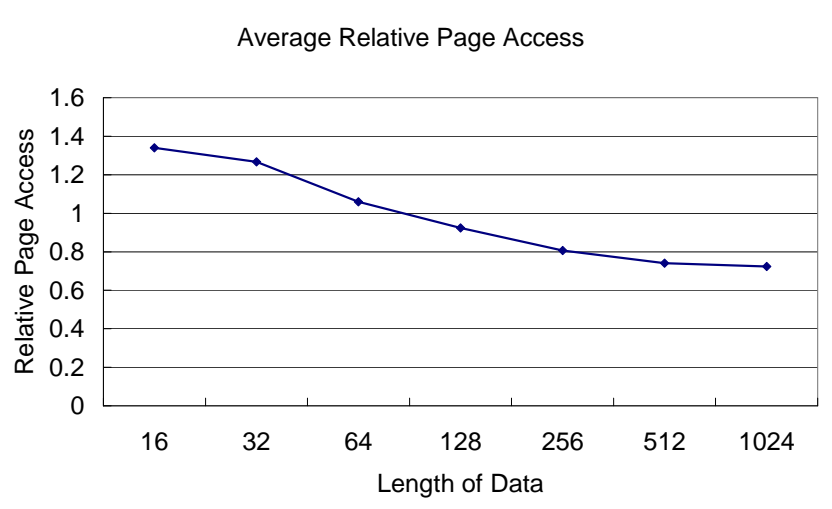

Figure 16: Average Relative Page Access vs. Length of Data

less-than-one relative page access at length 1024 backed up the above claim.

Moreover, Figure 15 shows that as much as half of the area in the graph is occupied by the lines representing different datasets. We can also readily see that the choice of datasets does make a big different, as also revealed in [12, 24]. Furthermore, although a generally decreasing trend can be observed, the rate of the decrease is quite different across different datasets, as visualized by the evenly distributed lines on the graph. This phenomenon is a sign that the properties of the data is a major determining factor on the performance of the index. Nevertheless, we chose to run the experiments on all 41 datasets instead of "cherry picking" only the datasets that work very well for the proposed algorithm. Our extensive experiments on the index using these various datasets from different domains have also demonstrated that improvements can be seen in at least half of the datasets and more improvements can be expected as the length of data and the number of data increase.

To reiterate, extensive experiments based on datasets collected across various domains demostrated the effectiveness of the index. And the effectiveness of the index is expected to improve as the length of data and the number of data increase.

Figure 17 shows the actual running time of the range queries as calculated by the difference between two calls to gettimeofday before and after the queries. The time is averaged over all 50 queries performed for each length of data of each dataset. It shows that the query generally runs very fast. All queries completed within a fraction of a second for all datasets of length 16 and 32, and all queries completed in the magnitude of minutes. Note the logarithmic scale in both axes.

The large differences in running time between different datasets are, again, a sign that the properties of the datasets significantly affect the performance of an index. However, we noted that, even for data of length 1024, most queries completed within five minutes, as can be seen in Figure 18.

Figure 18 shows the running time averaged over all datasets. It suggested that most queries actually completed well within a minute, even for the larger length of data. And even for

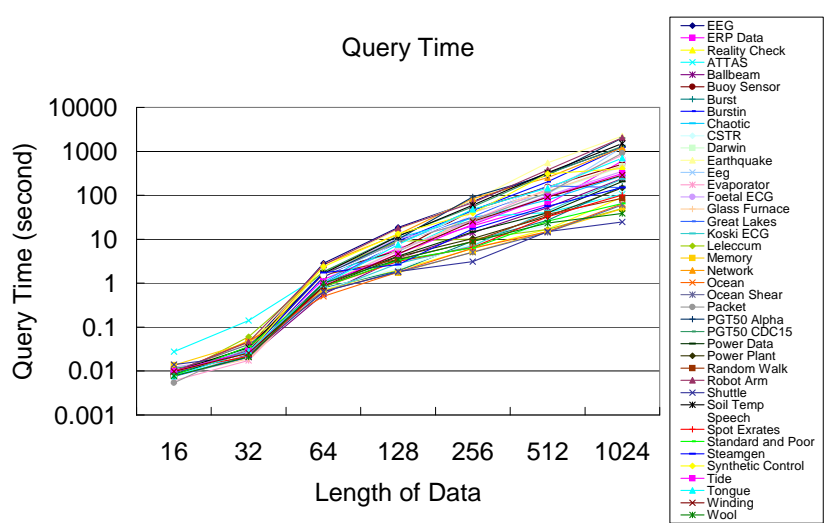

Figure 17: Query Time vs. Length of Data

the largest length of data, queries completed in 560 seconds on average. Recall from Figure 16 that linear scan perform better for datasets of shorter lengths; however, as Figure 18 shows, the query time for those datasets is actually not significant anyway. Moreover, the proposed index can significantly reduce the query time for datasets of longer lengths. Combining both advantages, our proposed index is capable as an all-round solution suitable for datasets of all lengths.

Average Query Time

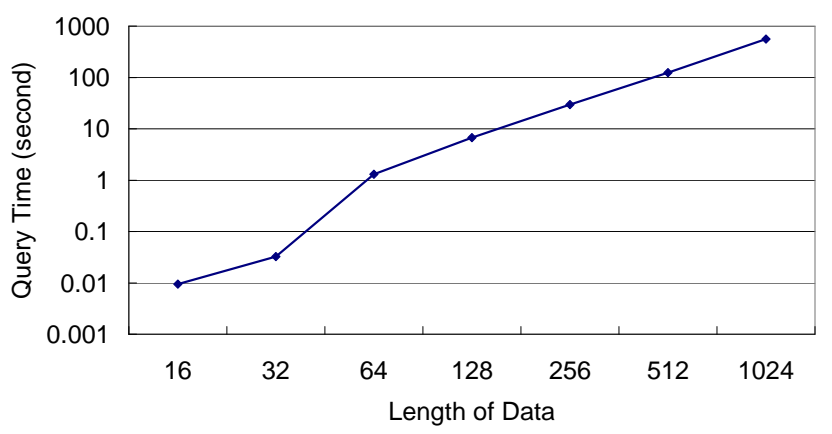

Figure 18: Average Query Time vs. Length of Data

Remarks: The mechanisms that we proposed can also help in Problem 3 in Section 2: Finding the best match to $Q$ in a database, for any rescaled version of $Q$, under the squared Euclidean distance. While this problem has been investigated before in [14], our modification reduces the size of the index by half and allows the user to specify the range of scaling without modifying the index. The size of index is cut by half because instead of indexing on bounding boxes (each box requires 2 vectors for the two boundaries for each dimension), we index on the time series (with 1 vector for each series).

\section{CONCLUSION}


We show the importance of the problem of SWM: handling scaling and time-warping distance in time series querying. To handle this problem, we propose a lower bound technique for pruning the search space. We show by experiment that we can typically prune over $90 \%$ of the search space. We also investigate into the problem of subsequence matching with SWM for which we device a indexing method and corresponding querying algorithm. We show by experiment that this method has increasing advantage over linear search when the length of data increases.

\section{REFERENCES}

[1] N. Beckmann, H.-P. Kriegel, R. Schneider, and B. Seeger. The $R^{*}$-tree: An efficient and robust access method for points and rectangles. In Proceedings of the 1990 ACM SIGMOD International Conference on Management of Data, pages 322-331, Atlantic City, New Jersey, United States, May 1990.

[2] S. Berchtold, D. A. Keim, and H.-P. Kriegel. The $\mathrm{X}$-tree: An index structure for high-dimensional data. VLDB'96, Proceedings of 22th International Conference on Very Large Data Bases, pages 28-39, Mumbai, India, Sept. 1996.

[3] L. Campbell and A. Bobick. Recognition of human body motion using phase space constraints. In Proceedings of International Conference on Computer Vision, pages 624-630, 1995.

[4] W. Chai and B. Vercoe. Folk music classication using hidden markov models. In Proceedings of International Conference on Articial Intelligence, 2001.

[5] C.Moeller-Levet, F.Klawonn, K.-H.Cho, and O.Wolkenhauer. Fuzzy clustering of short time series and unevenly distributed sampling points. In Proceedings of IDA, August 2003.

[6] N. Dalal and R. Horaud. Indexing key positions between multiple videos. In Proceedings of IEEE Workshop on Motion and Video Computing, pages 65-71, Orlando, FL, Dec. 2002.

[7] C. Faloutsos, M. Ranganathan, and Y. Manolopoulos. Fast subsequence matching in time-series databases. In Proceedings of the 1994 ACM SIGMOD International Conference on Management of Data, pages 419-429, Minneapolis, Minnesota, United States, May 1994.

[8] A. Guttman. R-trees: A dynamic index structure for spatial searching. In Proceedings of the 1984 ACM SIGMOD International Conference on Management of Data, pages 47-57, Boston, Massachusetts, June 1984.

[9] F. Itakura. Minimum prediction residual principle applied to speech recognition. IEEE Transactions on Acoustics, Speech, and Signal Processing, 23(1):67-72, Feb. 1975.

[10] A. Kale, R. Chowdhury, and R. Chellappa. Towards a view invariant gait recognition algorithm. In Proceedings of the IEEE International Conference on Advanced Video and Signal based Surveillance (AVSS), 2003.

[11] A. Kale, N. Cuntoor, B. Yegnanarayana, A. Rajagopalan, and R. Chellappa. Gait analysis for human identification. In Proceedings of the $3 r d$ International conference on Audio and Video Based Person Authentication, 2003.
[12] E. Keogh. Exact indexing of dynamic time warping. In $V L D B$ 2002, Proceedings of 28th International Conference on Very Large Data Bases, pages 406-417, Hong Kong, China, Aug. 2002.

[13] E. Keogh and T. Folias. The UCR Time Series Data Mining Archive. Available at http://www.cs.ucr.edu/ eamonn/TSDMA/index.html, 2002. Riverside CA. University of California Computer Science \& Engineering Department.

[14] E. Keogh, T. Palpanas, V. B. Zordan, D. Gunopulos, and M. Cardle. Indexing large human-motion databases. In Proceedings of 30th VLDB, 2004.

[15] N. Kosugi, Y. Sakurai, and M. Morimoto. Soundcompass: A practical query-by-humming system. In Proceedings of SIGMOD Conference, pages 881-886, 2004.

[16] G. Matessi, A. Pilastro, and G. Marin. Variation in quantitative properties of song among European populations of the reed bunting (Emberiza schoeniclus) with respect to bill morphology. Can. J. Zool, 78:428-437, 2000.

[17] C. Meek and W. Birmingham. The dangers of parsimony in query-by-humming applications. In Proceedings of International Symposium on Music Information Retrieval, 2003.

[18] Y.-S. Moon, K.-Y. Whang, and W.-K. Loh. Efficient time-series subsequence matching using duality in constructing windows. Technical Report 00-11-001, Advanced Information Technology Research Center (AITrc), KAIST, Taejon, Korea, Jan. 2000. http://aitrc.kaist.ac.kr/research/search.html.

[19] Y.-S. Moon, K.-Y. Whang, and W.-K. Loh. Duality-based subsequence matching in time-series databases. In Proceedings of the 17th International Conference on Data Engineering, pages 263-272, Heidelberg, Germany, Apr. 2001. IEEE.

[20] K. Pullen and C. Bregler. Motion capture assisted animation: Texturing and synthesis. $A C M$ Transactions on Graphics (Proc SIGGRAPH 2002), 22(3), July 2002.

[21] C. A. Ratanamahatana and E. Keogh. Everything you know about dynamic time warping is wrong. In Third Workshop on Mining Temporal and Sequential Data, Seattle, WA, Aug. 2004.

[22] H. Sakoe and S. Chiba. Dynamic programming algorithm optimization for spoken word recognition. IEEE Transactions on Acoustics, Speech, and Signal Processing, 26(1):43-49, Feb. 1978.

[23] B.-K. Yi, H. V. Jagadish, and C. Faloutsos. Efficient retrieval of similar time sequences under time warping. In Proceedings of the 14th International Conference on Data Engineering, Orlando, Florida, Feb. 1998. IEEE.

[24] Y. Zhu and D. Shasha. Warping indexes with envelope transforms for query by humming. In Proceedings of the 2003 ACM SIGMOD. 